\title{
EXPERIMENTAL PERFORMANCE INVESTIGATION OF DIGITAL BEAMFORMING ON SYNTHETIC APERTURE RADAR
}

\author{
Junghyo Kim ${ }^{1}$, Marwan Younis ${ }^{2}$, Werner Wiesbeck ${ }^{1}$ \\ ${ }^{1}$ University of Karlsruhe \\ Institut für Höchstfrequenztechnik und Elektronik \\ Kaiserstraße 12. 76131 Karlsruhe Germany \\ ${ }^{2}$ German Aerospace Center (DLR) \\ Microwaves and Radar Institute \\ P.O. box 1116, 82234 Wessling Germany
}

\begin{abstract}
In this paper, we present the experimental results of a Digital Beam Forming (DBF) Synthetic Aperture Radar (SAR) performance on the purpose of the HighResolution Wide-Swath (HRWS) SAR concept. A ground-based SAR system successfully demonstrated the DBF SAR operation. The demonstrator acquired SAR raw data with very dense spatial sampling rate in order to obtain various sampling rates. We evaluate DBF performance with respect to the image quality factor with two different types of the beam former, a fixed-beam former and an adaptive beam former. The results show that an adaptive DBF algorithm offers a wide range of the selection of the pulse repetition frequency (PRF). In addition we evaluate the noise performance compared to a reference mono-static SAR system on the same condition on single target experiment.
\end{abstract}

Index Terms - digital beamforming, ground-based demonstrator, SAR, HRWS SAR

\section{INTRODUCTION}

A spaceborne SAR system is used in the wide spectrum of the remote sensing owing to its wide-area observation capability and high resolution. A modern SAR system based on the phased-array antennas realizes the multiple SAR operation such as the stripmap, spotlight and scan mode operation. However, this system does not fulfill the users demands for SAR data for a wide area with the fine resolution since there is a fundamental trade-off between the ambiguity and the swath width. Additionally, the lifetime of a satellite depends on the fuel budget to maintain its orbit and motion control. A SAR sensor observing the wide area can reduce the revisit time and thereby extend the operation period. Therefore, the simultaneous achievement of the wide coverage and fine spatial resolution is quite attractive not only for the SAR users, but also in the system point of view. Such a simultaneous observation of a wide area with fine resolution provides the useful information, especially for the dynamic target surveillance of oceans, ice and artificial moving targets. On a bi- or multi-static configuration this fundamental restriction can be resolved by introducing an appropriate DBF technique. The HRWS SAR concept exploits the smart antenna technique on 2 dimensional array antenna constellation in order to compensate the azimuth ambiguity resulting from reduced PRF. In this paper we focus on the DBF SAR performance in azimuth direction. Several DBF algorithms were proposed with respect to a spectral estimation in spatial frequency domain [1], [2], [3]. In this paper, we introduce the experimental results through a simplified 2-dimensional measurement. Numerous array configurations are feasible for the experiment. Nevertheless, the preliminary experiment contributes to the evaluation of a uniform linear array (ULA) DBF SAR as shown in figure 1 .

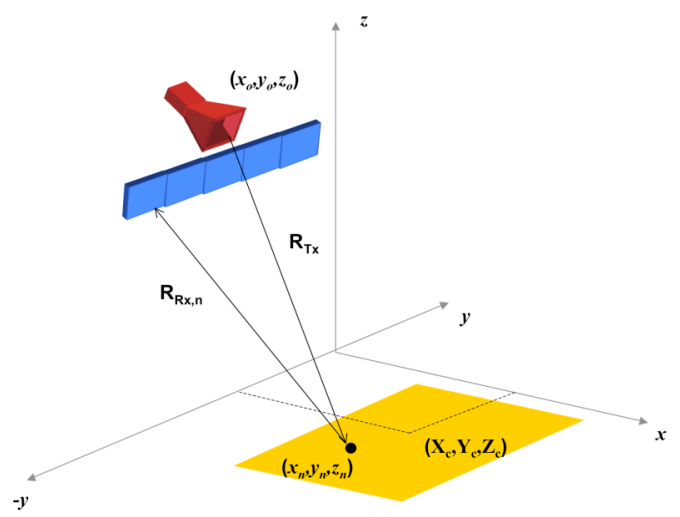

Figure 1. DBF SAR with ULA in along track

\section{GB DBF SAR DEMONSTRATION}

The measurement will be performed with the groundbased SAR system at X-band (center frequency: 9.65 $\mathrm{GHz}$ with $300 \mathrm{MHz}$ system bandwidth. The 
demonstration system is composed of the antenna subsystem, Radio Frequency (RF) subsystem and the platform block.

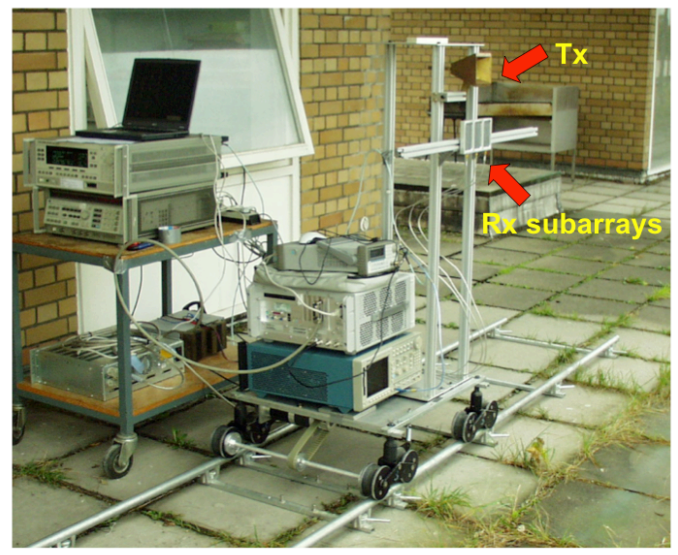

Figure 2. Photo of GB DBF SAR demonstrator

The implemented demonstrator contributes a single target measurement in order to verify the idea of DBF for the HRWS SAR concept and study the system features. A ULA with three receive sub-arrays are adapted and are vertically separated with $23 \mathrm{~cm}$ on purpose of avoiding coupling from the transmit antenna. The length of the receive sub-array is $6.8 \mathrm{~cm}$, which yields total length of $20.4 \mathrm{~cm}$. It should be noted that, in uniform sampling scenario, the separation between the effective phase centers corresponds to the half of the sub-array length. Hence the uniform spatial sampling fully depends on the antenna size [4], for the optimum sampling step movement of the platform must be $10.2 \mathrm{~cm}$. However, we evaluate the DBF SAR performance for various spatial sampling distance $\Delta \boldsymbol{d}$. The spatial sampling distance changes from $1.02 \mathrm{~cm}$ to $10.2 \mathrm{~cm}$ as shown in figure 3 .

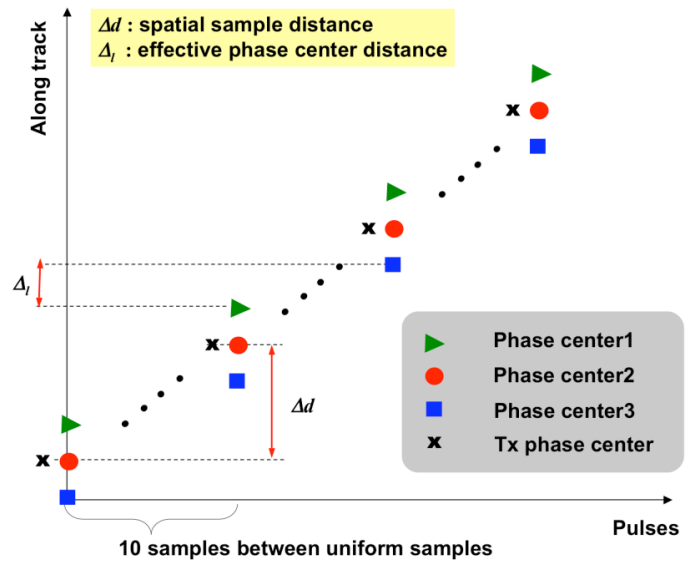

Figure 3. Spatial sampling scenario in GB experiments

\section{DBF SAR PERFORMANCE}

The obtained SAR data with DBF SAR operation is used to reconstruct the single point target response. The $\omega-k$ SAR processing algorithm is employed for the image reconstruction.

\subsection{Channel Imbalance Effect}

Prior to applying DBF on the raw data, the array system must be carefully calibrated. The former research shows that the channel imbalance causes a high frequency sinusoidal error. The critical point is the fact that the sinusoidal phase error has an identical frequency to PRF. This phase error hence causes unexpected spectral overlapping between original spectrum and shifted spectrum caused by the convolution relation in spatial Doppler domain. Usually array phase error can be easily corrected by the array calibration on the ground. However, the channel imbalance that occurs during the operation in the extreme environment should be considered in practice. Figure 4 shows the distorted target response by the high ambiguities that are generated by the channel imbalance.
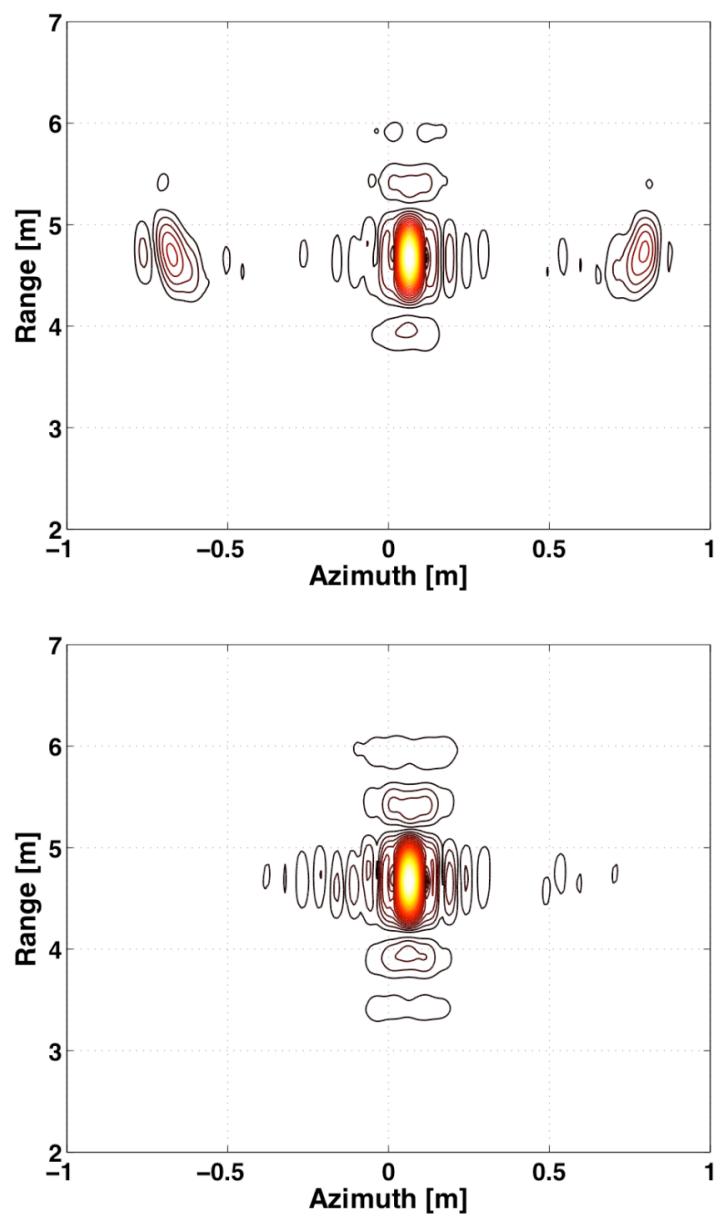

Figure 4. Contour plot of a point target before calibration (upper) and after calibration (lower)

\subsection{Non-uniform sampling effect}


After the array calibration we applied several DBF algorithms and reconstructed the point target response with various PRF. In this paper we consider a multiple orthogonal beam former using Discrete Fourier Transform (DFT) method and Minimum Variance Distortionless Response (MVDR) that is well known as Capon's beam former. We define the over-sampling ratio with respect to the optimum uniform sampling distance. In this experiment the uniform sampling distance is $10.2 \mathrm{~cm}$. If this optimum sampling distance is not fulfilled, then the raw data is acquired with non-uniform sampling in the azimuth. In principle the non-uniform sampling in the DBF SAR brings the same effect as the channel imbalance. However, it will be shown that the MVDR method and the reconstruction filter method provide the stable performance under the non-uniform condition.
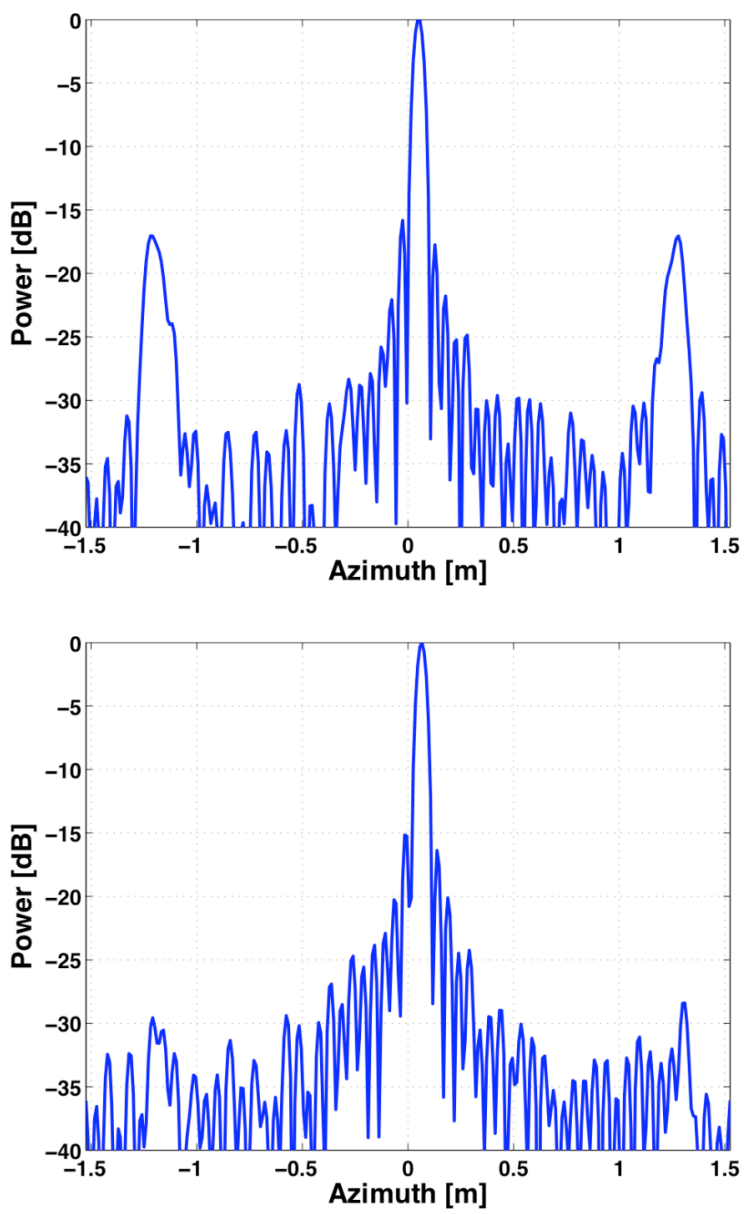

Figure 5. Azimuth profile with 50\% over-sampling ratio (upper) conventional orthogonal beam former and (lower) Capon's beam former

As shown in the right column of figure 5, the Capon's beam former provides the steady ambiguity suppression compared to the conventional fixed beam former in the same condition. We don't present the results of the reconstruction algorithm in [1] since the reconstruction filter algorithm shows an analogue of MVDR as investigated in [5]. Remarkable feature is the fact that the adaptive beam former, such as MVDR and reconstruction filter algorithm, adjusts the beam forming gain for the purpose of the adaptive null in non-uniform sampling. Thus the ideal Signal-to-Noise Ratio (SNR) is not achievable. It must be emphasized that the maximum sampling distance for a conventional mono-static SAR is $3.4 \mathrm{~cm}$ in this case. In the following section we analyze the more detail.

\section{ANALYSIS}

The reconstructed point target response is evaluated for the various spatial sampling rates, i.e. PRF, as introduced in previous section. In this section, we analyze the azimuth ambiguity suppression performance of the two beam formers. For the purpose we define peak-to-side lobe ratio (PSLR) that represent the ratio between main lobe level and the largest ambiguity level.

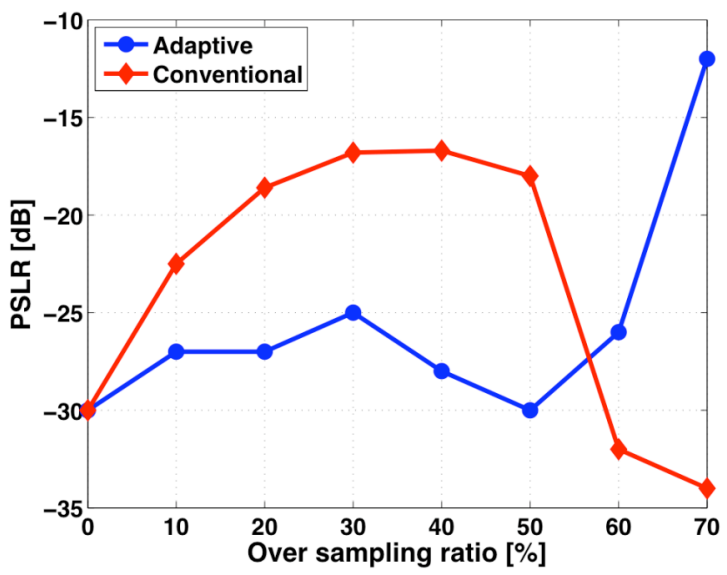

Figure 6. The measured Peak-to-Side Lobe Ratio (PSLR) with the over-sampling ratio

The "Adaptive" in the legend of figure 6 stands for the Capon's MVDR beam former and the "Conventional" means the orthogonal beam former. It is obvious that the Capon's beam former shows the stable suppression property compared to the fixed beam former. In the higher over-sampling region around $66 \%$ a breakdown point exists. The reconstruction filter algorithm and Capon's algorithm take a matrix inverse to define the weight matrix. Around the breakdown point the covariance matrix becomes singular due to the coincident spatial samples. Thus a proper weight matrix cannot be defined. In contrast to the adaptive beam former, the fixed beam former has second null point at this point. So the performance becomes similar to the uniform sampling case with this sampling ratio. These results can be observed in the Integrated Side Lobe Ratio (ISLR) as shown in figure 7. Although it doesn't show the pure noise performance of only the beam former, the ISLR reflects 
the estimated noise performance of the DBF SAR. As shown above the non-uniform sampling affects the total noise on the SAR image in case of the conventional beam former compared to the image from the adaptive beam former. At the break point the ISLR of the adaptive beam former degrade significantly due to the same effect stated above.

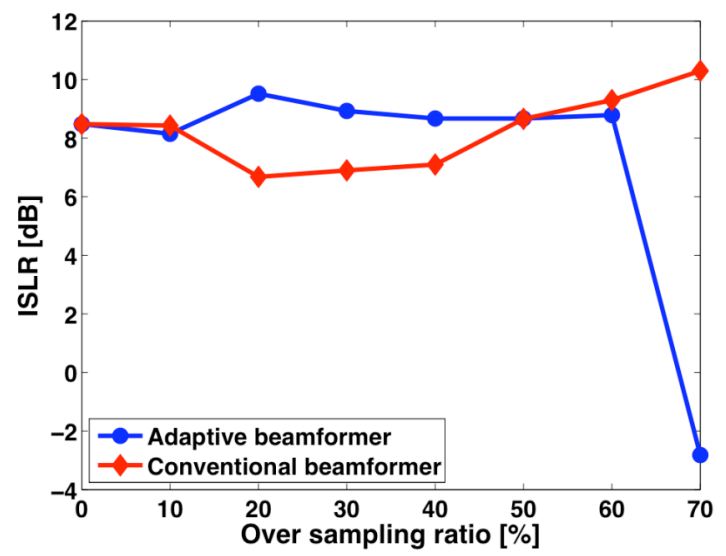

Figure 7. The measured Integrated Side Lobe Ratio (ISLR) with the over-sampling ratio

\section{SUMMARY}

Using a ground-based SAR system we evaluate the proposed DBF performance for SAR application. It is obviously demonstrated that the DBF SAR allows increasing the swath width and provides the performance improvement. In practice, we could observe the fact that an accurate array calibration is necessary to achieve the optimum performance. The effect of systematic phase and amplitude error is servere because it contributes unexpected spectral overlapping. From the various spatial sampling experiments we showed that the adaptive beamformer has tolerance to select the wide range of PRF. However, it is emphasized that the adaptive beam former fully dependent to the SNR of the input signal and calibration accuracy. Therefore an optimization strategy in practical system design will be studied in future work.

\section{REFERENCES}

[1] G. Krieger, N. Gebert, and A. Moreira, "Unambiguous SAR Signal Reconstruction from Non-Uniform Displaced Phase Centre Sampling," IEEE GRS Letters, Vol. 1, No. 4, pp. 260264, Oct. 2004.

[2] M. Younis, Digital Beamforming for High Resolution Wide Swath Real and Synthetic Aperture Radar, Ph.D. Dissertation, IHE, Universität Karlsruhe, 2004.

[3] Z. Li, H. Wang, and Z. Bao, "Generation of Wide-Swath and High-Resolution SAR Images from Multichannel Small Spaceborne SAR System," IEEE GRS Letters, Vol. 2, No. 1, pp. 82-86, Jan. 2005.

[4] M. Younis, C. Fischer, and W. Wiesbeck, "Digital Beam Forming in SAR System," IEEE Transaction on GRS, Vol. 41, No. 71, pp. 1735-1739, Jul. 2003.

[5] N. Gebert, G. Krieger, and A. Moreira, "Digital Beam Forming for HRWS-SAR Imaging: System Design, Performance, and Optimization Strategies," IEEE Proceeding IGARSS, pp. 1836-1839, Jul. 2006. 